$110 \begin{aligned} & \text { PRÓ-REITORIA } \\ & \text { DE EXTENSÃO } \\ & \text { E CULTURA }\end{aligned}$

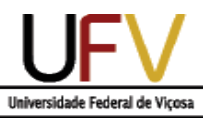

www.periodicos.ufv.br/elo

elo@ufv.br

ISSN: $2317-5451$

\title{
Segurança alimentar de agricultores agroecológicos do Semiárido Brasileiro
}

\author{
José Vagner Silval, Vanessa Schiavon Lopes ${ }^{2}$, Maria Valdenira Rodrigues de \\ Almeida ${ }^{3}$, Ana Leônia de Araújo Girão ${ }^{4}$, Pollyanna da Silva Quemel ${ }^{5}$, Renato \\ Teixeira de Oliveira ${ }^{6}$, Teogenes Senna de Oliveira ${ }^{7}$
}

Resumo: O presente estudo buscou descrever a situação de segurança alimentar vivenciada por famílias de agricultores agroecológicos dos municípios de Choró, Massapê e Tauá, assistidos pela Organização Não Governamental ESPLAR no Ceará. Utilizou-se a Escala Brasileira de Insegurança Alimentar (EBIA), que tem o fator renda como principal determinante e a EBIA adaptada que leva em consideração a produção para autoconsumo. Foram entrevistadas 61 famílias de agricultores agroecológicos que continham em seu núcleo pelo menos um membro menor de dezoito anos e outra entrevista foi realizada com famílias em que todos os membros eram maiores de 18 anos. Os resultados sugerem que o fator renda não foi o único determinante na segurança alimentar e nutricional desses agricultores, enfatizando a importância da produção para autoconsumo. A verificação da insegurança alimentar pela EBIA pode ser superestimada por não considerar a produção para o autoconsumo. Os métodos agroecológicos combinados com a troca de conhecimentos podem garantir a segurança alimentar

Palavras-chave: Agricultura familiar. Consórcios agroecológicos. Segurança alimentar.

Área Temática: TSegurança Alimentar.

\section{Food security of agricultural farmers in the Brazilian Semiarid}

\begin{abstract}
The present study sought to describe the food security situation experienced by families of agroecological farmers in the municipalities of Choró, Massapê and Tauá, assisted by the Non-Governmental Organization ESPLAR in Ceará. The Brazilian Food Insecurity Scale (EBIA) was used, which has the income factor as the main determinant and the adapted EBIA that takes into account production for self-consumption. 61 families of agroecological farmers were interviewed, which contained at least one member under eighteen years old and another interview was made with families that had all members over 18 years old. The results suggest that the income factor was not the only determinant in the food and nutritional security of these farmers, emphasizing the importance of production for self-consumption. The verification of food insecurity by EBIA can be overestimated for not considering production for self-consumption. Agroecological methods combined with the exchange of knowledge can guarantee food security.
\end{abstract}

Keywords: Familiar agriculture. Agroecological intercropping. Food security.

\footnotetext{
${ }^{1}$ Doutor, Professor EBTT do Instituto Federal de Educação, Ciência e Tecnologia de Rondônia, Campus Colorado do Oeste. Endereço: BR 435, km 63, Zona Rural, 76993-000, Colorado do Oeste -RO, Brasil. Cel: 69 99256-2346, e-mail:vagner.silva@ifro.edu.br

${ }^{2}$ Doutora em Solos e Nutrição de Plantas pela Universidade Federal de Viçosa, Campus Viçosa (MG), Brasil. E-mail: vanessaschyavon@gmail.com

${ }^{3}$ Engenheira Agrônoma pela Universidade Federal do Ceará. Consultora na empresa V. Fair Trade. E-mail: val@ veja.fr.

${ }^{4}$ Engenheira Agrônoma pela Universidade Federal do Ceará. Fiscal Ambiental da Superintendência Estadual de Meio Ambiente do Ceará (SEMACE). E-mail: analeonia@yahoo.com.br

${ }^{5}$ Engenheira Agrônoma pela Universidade Federal do Ceará, Brasil. E-mail: agroquemel@ gmail.com

${ }^{6}$ Mestre em Fitotecnia pela Universidade Federal do Ceará, Brasil. E-mail: renato.agro@yahoo.com.br

${ }^{7}$ Doutor, Professor Titular da Universidade Federal de Viçosa, Campus Viçosa (MG), Brasil. E-mail: teo@ufv.br
} 


\section{Seguridad alimentaria de los agricultores agroecológicos en la región semiárida brasileña}

Resumen: El presente estudio buscó describir la situación de seguridad alimentaria que viven familias de agricultores agroecológicos en los municipios de Choró, Massapê y Tauá, asistidos por la Organización No Gubernamental ESPLAR en Ceará. Se utilizó la Escala Brasileña de Inseguridad Alimentaria (EBIA), que tiene el factor ingreso como principal determinante y la EBIA adaptada que toma en cuenta la producción para el autoconsumo. Se entrevistaron 61 familias de agricultores agroecológicos, que contenían al menos un miembro menor de dieciocho años y otra entrevista fue realizada con familias que conteníam todos los miembros mayores de 18 años. Los resultados sugieren que el factor ingreso no fue el único determinante en la seguridad alimentaria y nutricional de estos agricultores, enfatizando la importancia de la producción para el autoconsumo. La verificación de la inseguridad alimentaria por EBIA puede estar sobreestimada por no considerar la producción para el autoconsumo. Los métodos agroecológicos combinados con el intercambio de conocimientos pueden garantizar la seguridad alimentaria.

Palabras clave: Agricultura familiar. Consorcios agroecológicos. Seguridad alimentaria.

\section{INTRODUÇÃO}

O modelo agrícola convencional, centrado no uso predatório de recursos naturais e uso abusivo de agroquímicos, permitiu aumentar a produção e a produtividade de alguns cultivos em certas regiões. O que causa, no entanto, forte agressão ao ambiente e comprometimento da sua sustentabilidade a longo prazo. Este cenário reflete num modelo de agricultura voltado à exportação de commodities e altamente dependente de insumos químicos, trazendo riscos à saúde humana, ao meio ambiente, à segurança e soberania alimentar da população (BORGES, 2018).

A insegurança alimentar é um importante problema social e de saúde pública em todo o mundo (PALMEIRA, 2020). De acordo com as últimas estimativas, 9,2\% da população mundial (um pouco mais de 700 milhões de pessoas) foi exposta a graves níveis de insegurança alimentar em 2018 (FAO, 2019). A alta taxa de insegurança alimentar mundial tem sido atribuída aos seguintes fatores: pobreza, injustiça social, instabilidade política, conflitos armados, desaceleração do crescimento econômico, sistemas alimentares insustentáveis e insalubres e mudanças climáticas adversas (FAO, 2018).

No Brasil, foi diagnosticado que $22,6 \%$ da população brasileira ( \pm 50 milhões de pessoas) vive com algum nível de insegurança alimentar e a maior parte está localizada na região Nordeste do País (38,1\%), seguido do Norte (36,1\%), Centro-Oeste (18,2\%), Sul (14,9\%) e Sudeste (14,5\%) (IBGE, 2013). Cabe registrar que a incidência da fome, manifestação mais grave da insegurança alimentar, diminuiu na última década devido ao aumento da produção de alimentos. Porém, recentemente, a fome voltou a aumentar, afetando 815 milhões de pessoas em 2016 (PEREIRA; WYNBERG; REIS, 2018).

Novos desafios para segurança alimentar são impostos pelas mudanças climáticas, principalmente nos ambientes tropicais, onde essas mudanças podem resultar em eventos de chuva mais intensos entre períodos de seca prolongados (ROSEGRANT e CLINE, 2003). Tais condições podem promover pragas e doenças nas plantações e na pecuária, bem como no solo (ROSEGRANT e CLINE, 2003). 
Abordagens agroecológicas que buscam gerenciar paisagens para produção agrícola e serviços ecossistêmicos podem ser uma maneira de melhorar a produtividade das culturas, pois reduzem a poluição por meio de métodos alternativos no manejo de nutrientes e pragas, criam reservas de biodiversidade e aumentam qualidade do habitat através da prática cuidadosa do solo, da água e da vegetação natural (GLIESSMAN e TITTONELL, 2015; ROSEGRANT e CLINE, 2003). Ao construir sinergias, a agroecologia pode aumentar a produção de alimentos e a segurança alimentar e nutricional, enquanto restaura os serviços do ecossistema e a biodiversidade (GLIESSMAN e TITTONELL, 2015).

Inciativas que implicam no emprego da agroecologia por agricultores, movimentos sociais, organizações não governamentais, a exemplo da organização não governamental ESPLAR (ONG ESPLAR) no Ceará, dentre outras instituições, podem promover a segurança alimentar e nutricional, conservando os recursos naturais, a agrobiodiversidade e a conservação do solo e da água em comunidades rurais de várias regiões do Brasil.

Nessa perspectiva, demonstrar a validade e o potencial econômico da agroecologia é fundamental (PEREIRA; WYNBERG; REIS, 2018). Prova disso são as transformações ocorridas no Programa Nacional de Alimentação Escolar (PNAE) e na legislação, que sugerem a reeducação alimentar nas escolas públicas brasileiras com foco no consumo de alimentos saudáveis produzidos de forma agroecológica (NASCIMENTO et al., 2019).

A constatação, em muitos países, de que o rendimento domiciliar ou outros indicadores indiretos são insuûcientes para identiûcar populações sob o risco de insegurança alimentar, levou ao desenvolvimento de uma escala de medida direta da Insegurança Alimentar e Fome do Departamento de Agricultura dos Estados Unidos (United States Department of Agriculture - USDA) (BICKEL et al., 2000), possibilitando que essa condição seja mensurada de forma válida, confiável e direta (RADIMER et al., 1992).

A escala desenvolvida pela USDA tornou-se a base para realizar a estimativa oficial do número de pessoas em situação de insegurança alimentar nos EUA(BHATTACHARYA et al., 2004; MCKECHNIE et al., 2018), sendo utilizada por inúmeros pesquisadores (ALAIMO et al., 2001; BORJAS, 2002; WEINREB et al., 2002; ABOABA et al., 2020). Além disso, é utilizada para elaborar o diagnóstico da condição de segurança alimentar e indicar populações sob maior risco de insegurança, servindo, também, para observar o impacto de políticas públicas sobre a condição de acesso da população à alimentação adequada (IBGE, 2006).

AEscala Brasileira de Insegurança Alimentar- EBIAé uma adaptação da escala USDA para a realidade brasileira e é aplicada pelo Instituto Brasileiro de Geografia Estatística (IBGE) na Pesquisa Nacional por Amostras de Domicílios - PNAD de 2004, com o objetivo de realizar um diagnóstico sobre a situação da segurança alimentar nutricional no país. A escala tem sido utilizada por gestores públicos de todos os níveis, para identificar populações vulneráveis, avaliar e ajustar os seus programas e ações (SEGALL-CORRÊA; MARIN-LEON, 2009), bem como por pesquisadores da área.

Contudo, segundo Hoffmann (1995), a EBIA não considera o valor da produção para autoconsumo que é um componente importante da renda real para os pequenos agricultores. Dizer que uma pessoa passa fome apenas 
por não ter poder aquisitivo suficiente para comprar alimentos é uma análise limitada (Hoffmann, 1995), o que leva a necessidade de considerar o conceito de segurança alimentar e nutricional em uma perspectiva multidimensional.

Dessa forma, será possível vislumbrar outros determinantes, que juntamente à renda ou até mesmo independente dela, contribuem para garantir acesso aos alimentos. Sobre isso, Grisa (2011) cita a produção para autoconsumo como um dos diferentes tipos de recursos não monetários que possibilita o acesso à alimentação. Um exemplo desta condição são os agricultores familiares tradicionais que produzem para subsistência.

Há diversos estudos abordando os aspectos históricos, sociológicos, técnicos, ambientais e econômicos com os agricultores envolvidos nesse estudo. A exemplo, têm-se as pesquisas realizadas em Tauá que observaram os benefícios dos sistemas ecológicos para a qualidade do solo (OTUTUMI et al., 2004). No município de Choró, Aguiar (2008) averiguou os indicadores da qualidade física do solo em áreas de cultivo agroecológico; Almeida et al. (2015) pesquisou a biodiversidade e Cardoso (2017) analisou os aspectos da produção e comercialização. Contudo, especificamente sobre a segurança alimentar e nutricional, há escassez de resultados, o que aumenta a relevância dos dados.

\section{OBJETIVO}

Pressupondo que a produção agroecológica desenvolvida por agricultores do semiárido cearense contribui para a segurança alimentar e nutricional de suas famílias, desenvolveu-se o presente trabalho com intuito de avaliar a insegurança alimentar através da Escala Brasileira de Insegurança Alimentar (EBIA) na versão original e na adaptada (que desconsidera o fator renda), aplicada a agricultores familiares do semiárido cearense que conduzem consórcios agroecológicos.

\section{METODOLOGIA}

\section{Área de estudo e população}

A pesquisa foi realizada em 2007 na região semiárida do Ceará, mais precisamente nos municípios de Choró, Massapé e Tauá. O município de Choró está localizado na microrregião do Sertão de Quixeramobim, mesorregião dos Sertões Cearenses. Apresenta clima tropical quente semiárido, de acordo com classificação de Köppen, com temperaturas variando entre 26 e $28^{\circ} \mathrm{C}$ e precipitação pluviométrica média anual de $992 \mathrm{~mm}$ (IPECE, 2007). Massapê está localizada, microrregião de Sobral, mesorregião do Noroeste Cearense. O município apresenta três zonas climáticas: clima tropical quente úmido, clima tropical quente semiárido brando e clima tropical quente semiárido, apresentando temperaturas amenas, tendo seus valores máximos variando entre $26^{\circ}$ e $28^{\circ} \mathrm{C}$, com período chuvoso de janeiro a maio, com pluviosidade média de 749,5 mm (OLIVEIRA et al., 2019). Tauá fica situado no sudeste do Estado do Ceará, na microrregião geográfica Sertão dos Inhamuns, a $320 \mathrm{~km}$ da capital, Fortaleza. A classificação climática de Köppen é do tipo clima tropical quente semiárido, com temperaturas médias anuais em torno de $27,4^{\circ} \mathrm{C}$ e médias pluviométricas anuais de 483,53 $\mathrm{mm}$ (INMET, 2016). 
Após esses anos de diversos ciclos de cultivo de algodão na forma de consórcios agroecológicos com culturas alimentares, houve o aprimoramento das técnicas do sistema pelos agricultores, que contaram com a colaboração da pesquisa e assistência técnica da Empresa de Pesquisa Agropecuária de Minas Gerais (EMBRAPA) e do Projeto Dom Helder Camara (PDHC), possibilitando o aumento na produção do algodão e das outras culturas do consórcio. Esses agricultores atualmente estão organizados em três associações e possuem relacionamento com a OPAC (Organismos de Averiguação da Conformidade Orgânica), esquemas de comercialização justos, que agregam valor pelo processamento parcial do algodão produzido, além de certificação orgânica e entrada no mercado internacional.

Nos últimos anos houve um boom do mercado do algodão orgânico. Mudanças no mercado da moda aumentaram a procura por "matérias-primas sustentáveis", favorecendo esse nicho mercadológico e a consolidação das parcerias com as compradoras de algodão agroecológico. Tais fatores, possivelmente, contribuíram para o aumento da renda e ampliação da segurança alimentar dos agricultores, ao longo desses anos.

Os atores e parceiros importantes para a pesquisa e envolvidos na rede do algodão agroecológico no Ceará são instituições públicas (Embrapa, Prefeituras dos municípios envolvidos, Governo Federal, Governo Estadual, Ministério do Desenvolvimento Agrário (MDA), Universidade Federal do Ceará (UFC); organizações dos agricultores (Associação de Desenvolvimento Educacional e Cultural de Tauá - CE (ADEC); Associação de Certificação Participativa Agroecológica (ACEPA), Associação Agroecológica de Certificação Participativa dos Inhamuns/Crateús (ACEPI); Sindicato dos Trabalhadores e Trabalhadoras Rurais dos municípios de Choró, Quixeramobim, Quixadá, Tauá, Nova Russas e Tamboril; Organizações não governamentais e empresas: ESPLAR - Centro de Pesquisa e Assessoria, Veja Fair Trade (Vert), Cooperativa Central Justa Trama (Cardoso, 2017).

Oestudo abrangeu uma amostra de 61 famílias de agricultores, assistidos pela organização não governamental ESPLAR - Centro de Pesquisa e Assessoria. AONG e um grupo de famílias de agricultores deram início às práticas sustentáveis nos anos 90, os quais envolveram o uso de sistemas consorciados com algodão, milho, feijão, gergelim, leucena e guandu (MAIA et al., 2019). Na condução das áreas de cultivo, realizou-se o preparo do solo, o enleiramento de restos das culturas, a construção de cordões de pedra permanente em nível e capinas seletivas. A adubação constou da aplicação de biofertilizantes à base de esterco fresco, rapadura, água e outros componentes de origem animal (sangue + vísceras de animais domésticos), vegetal (folhas e ramos de plantas nativas trituradas e maceradas) e mineral (cinzas), fermentados durante 45 dias e aplicados nas fases vegetativa e reprodutiva das culturas, numa proporção de $1 \mathrm{~L}$ de solução para cada $10 \mathrm{~L}$ de água. Esses produtos foram aplicados, na forma de suspensão, com pulverizadores costais, em intervalos de uma a duas semanas.

Foram utilizadas ainda técnicas de controle de pragas e doenças como catação e destruição dos botões florais e maçãs de algodão atacados pelo bicudo do algodoeiro (Anthonomus grandis), assim como o uso de cultura armadilha - também denominado de "planta-isca", que baseia-se no plantio (antecipado ou não) de uma espécie mais atrativa (gergelim - Sesamum indicum) para as pragas (mosca branca e pulgão) do algodoeiro. Essa espécie hospedeira é plantada nas fileiras marginais do campo, visando estimular a praga em preterir ou 
retardar a colonização definitiva no algodoeiro e, assim, seriam controladas com produtos naturais, instalação de Tubo Mata Bicudo (TMB), contendo o feromônio grandlure, antes da semeadura e após a colheita. Foram realizadas aplicações do extrato natural das folhas de nim (Azadirachta indica) a $3 \%(\mathrm{~m} / \mathrm{v})$, no controle alternativo da vaquinha (Diabrotica speciosa e Cerotoma arcuata), presentes no feijão-de-corda, e da lagarta do cartucho (Spodoptera frugiperda), no milho; e o uso do agente de controle biológico Trichogramma spp. (para o controle de curuquerê, lagarta rosada e lagarta-das-maçãs). Todos os produtos dos consórcios fazem parte da cadeia de comercialização agroecológica que compõem forma de comércio justo (MAIA et al., 2019), com intuito de facilitar o acesso direto ao mercado em condições justas e equitativas para os pequenos agricultores, criando um canal de comercialização sustentável, solidário e de qualidade.

\section{Seleção de agricultores familiares e obtenção dos dados}

O cultivo de algodão em bases agroecológicas teve início no Ceará, no município de Tauá nos anos 90 e estendeu-se para outros municípios do Estado. Ao longo dos anos, houve variação no número de agricultores envolvidos na proposta agroecológica. Até o ano de coleta dos dados havia ocorrido três fases. $\left.1^{\text {a }}\right) 1993$ a 1997; $\left.2^{\text {a }}\right) 1998$ a 2000; 3ª 2001 a 2008; com adesões e saídas de agricultores. Daí o critério de seleção de agricultores participantes da proposta agroecológica nos 3 municípios estudados. O grupo de agricultores entrevistados possuía no mínimo três anos de inserção na proposta agroecológica e participaram ao longo desse período de eventos formativos sobre agroecologia e segurança alimentar, além de formação teórica e prática através da realização da transição agroecológica do seu sistema produtivo. (Ver mais em: https://esplar.com.br/).

Para realização da pesquisa foram utilizados dados primários obtidos por meio de entrevistas diretas junto aos agricultores familiares, cadastrados no ESPLAR. O número total de famílias envolvidas e aquelas entrevistadas por município encontra-se na Tabela 1.

Tabela 1 -Número de agricultores da populaçãoe da amostra selecionada para avaliação por município estudado, 2007.

\begin{tabular}{|c|c|c|}
\hline Municípios & $\begin{array}{c}\text { Número total de agricultores } \\
\text { familiares }\end{array}$ & $\begin{array}{c}\text { Número de agricultores familiares } \\
\text { selecionados }\end{array}$ \\
\hline Choró & 23 & 18 \\
\hline Massapé & 33 & 17 \\
\hline Tauá & 60 & 26 \\
\hline Total & 116 & 61 \\
\hline
\end{tabular}

Fonte: Autoria própria.

\section{Variáveis econômicas}

Nos três municípios estudados, por meio de questionários aplicados em entrevistas com os agricultores familiares, foram obtidas informações de caráter econômico, tais como: renda familiar mensal e per capita, importantes indicadores para avaliação da insegurança alimentar. 


\section{Avaliação da segurança alimentar}

Para averiguar a situação de segurança alimentar dos agricultores, recorreu-se a EBIA, que consiste em uma escala de medida direta dessa condição, composta por 15 perguntas com respostas de sim ou não (SEGALLCORREAA; MARIN-LEON, 2009) e cuja síntese encontra-se na Tabela 2. Todas as perguntas referem-se aos três meses que antecederam às entrevistas.

A EBIA permite a classificação das famílias ou dos domicílios em quatro categorias: segurança alimentarquando não há restrição alimentar de qualquer natureza, nem mesmo a preocupação com a falta de alimentos no futuro; insegurança alimentar leve - quando há preocupação ou incerteza quanto ao acesso aos alimentos, portanto, risco para a sustentabilidade e, ainda, comprometimento da qualidade da dieta; insegurança alimentar moderadaquando aparecem restrições quantitativas especialmente relevantes entre pessoas adultas; e insegurança grave quando há redução importante da quantidade de alimentos disponíveis, tanto para a alimentação de pessoas adultas como para a de crianças que residem no domicílio (SEGALL-CORRÊA; MARIN-LEON, 2009).

Tabela 2 - Questões da Escala de Insegurança Alimentar (EBIA) aplicadas aos agricultores familiares agroecológicos do semiárido cearense.

1. Moradores tiveram preocupação de que os alimentos acabassem antes de poderem comprar ou receber mais comida?

2. Alimentos acabaram antes que os moradores tivessem dinheiro para comprar mais comida?

3. Moradores ficaram sem dinheiro para ter uma alimentação saudável e variada?

4. Moradores comeram apenas alguns alimentos que ainda tinham por que o dinheiro acabou?

5. Algum morador de 18 anos ou mais de idade diminuiu alguma vez a quantidade de alimentos nas refeições ou deixou de fazer alguma refeição porque não havia dinheiro para comprar comida?

6. Algum morador de 18 anos ou mais de idade alguma vez comeu menos porque não havia dinheiro para comprar comida?

7. Algum morador de 18 anos ou mais de idade alguma vez sentiu fome, mas não comeu porque não havia dinheiro para comprar comida?

8. Algum morador de 18 anos ou mais de idade perdeu peso porque não comeu quantidade suficiente de comida devido à falta de dinheiro para comprar comida?

9. Algum morador de 18 anos ou mais de idade alguma vez fez apenas uma refeição ou ficou um dia inteiro sem comer porque não havia dinheiro para comprar comida?

10. Algum morador com menos de 18 anos de idade alguma vez deixou de ter uma alimentação saudável e variada porque não havia dinheiro para comprar comida?

11. Algum morador com menos de 18 anos de idade alguma vez não comeu quantidade suficiente de comida porque não havia dinheiro para comprar comida?

12. Algum morador com menos de 18 anos de idade diminuiu a quantidade de alimentos nas refeições porque não havia dinheiro para comprar comida?

13. Algum morador com menos de 18 anos de idade alguma vez deixou de fazer uma refeição porque não havia dinheiro para comprar comida?

14. Algum morador com menos de 18 anos de idade alguma vez sentiu fome, mas não comeu porque não havia dinheiro para comprar comida?

15. Algum morador com menos de 18 anos de idade alguma vez ficou um dia inteiro sem comer porque não havia dinheiro para comprar comida?

Fonte: (SEGALL-CORRÊA; MARIN-LEON, 2009). 
Para a classificação da segurança ou insegurança alimentar das famílias são atribuídas pontuações com a soma de valor 1 (um) para cada resposta positiva e caso a resposta seja negativa, nenhum ponto é acrescentado (VILAS BOAS, 2017). Ao final do questionário, quanto mais pontos o indivíduo obtiver, maior é o quadro de insegurança alimentar, havendo uma classificação para cada pontuação alcançada na escala (Tabela 3).

Tabela 3 - Classificação de insegurança alimentar dos domicílios com base na Escala de Insegurança Alimentar (EBIA).

\begin{tabular}{|l|c|c|}
\hline \multirow{2}{*}{ Classificação } & \multicolumn{2}{|c|}{$\mathbf{N}^{\mathbf{0}}$ de respostas positivas } \\
\cline { 2 - 3 } & $\begin{array}{c}\text { (Famílias com menores } \\
\text { de 18 anos) }\end{array}$ & $\begin{array}{c}\text { (Famílias sem menores } \\
\text { de 18 anos) }\end{array}$ \\
\hline Segurança alimentar & 0 & 0 \\
\hline Insegurança leve & 1 a 5 & 1 a 3 \\
\hline Insegurança moderada & 6 a 10 & 4 a 6 \\
\hline Insegurança grave & 11 a 15 & 7 a 9 \\
\hline
\end{tabular}

Fonte: (SEGALL-CORRÊA; MARIN-LEON, 2009).

Para verificar se a adoção do sistema de produção agroecológico pelos agricultores dos municípios estudados contribuiu para a situação de segurança alimentar desses agricultores, foi utilizada uma escala na versão adaptada (que desconsidera o fator renda) aplicadas aos agricultores familiares do semiárido cearense que conduzem os consórcios agroecológicos.

Tabela 4 - Questões da Escala de Insegurança Alimentar adaptada da EBIA aplicadas aos agricultores familiares agroecológicos do semiárido cearense.

1. Os moradores tiveram preocupação que os alimentos produzidos acabassem antes de ter como conseguir mais alimentos?

2. Os alimentos produzidos acabaram antes que os moradores pudessem conseguir mais alimentos?

3. Moradores ficaram sem uma alimentação saudável e variada?

4. Moradores comeram apenas alguns alimentos que ainda tinham em casa?

5. Algum morador de 18 anos ou mais, diminuiu alguma vez a quantidade de alimentos nas refeições ou deixou de fazer alguma refeição por que não havia maior quantidade de alimento?

6. Algum morador de 18 anos ou mais, comeu menos porque a quantidade de alimentos não daria para todos se comesse a quantidade de costume?

7. Algum morador de 18 anos ou mais, sentiu fome e não comeu porque a quantidade de alimentos estava reduzida?

8. Algum morador de 18 anos ou mais, emagreceu porque a quantidade de alimentos armazenados estava muito reduzida?

9. A Algum morador com menos de 18 anos deixou de ter uma alimentação saudável e variada porque estava sem alimentos armazenados? 
Tabela 4 - Cont.

10. Algum morador com menos de 18 anos, não comeu quantidade suficiente de comida porque estava sem alimentos armazenados?

11. Algum morador com menos de 18 anos diminuiu a alimentação porque a quantidade de alimentos não daria para todos, se comesse quantidade de costume?

12. Algum morador com menos de 18 anos sentiu fome e não comeu porque a quantidade de alimentos estava reduzida?

13. Algum morador com menos de 18 anos emagreceu porque a quantidade de alimentos armazenados estava muito reduzida?

14. Algum morador com menos de 18 anos emagreceu porque a quantidade de alimentos armazenados estava muito reduzida?

15. Algum morador com menos de 18 anos, alguma vez ficou o dia inteiro sem comer porque não tinha comida?

\section{RESULTADOS E DISCUSSÃO}

Na aplicação da EBIA constatou-se que mais de $80 \%$ das famílias de agricultores agroecológicos com ao menos um membro menor de 18 anos de idade do município de Choró, se referiram a algum grau de insuficiência alimentar nos três meses anteriores às entrevistas, conforme mostra a Figura $1 \mathrm{~A}$. Dessas, $31 \%$ conviveram no período de um mês, com a experiência de passar fome em ao menos um dia nesse período (Figura 1A). A partir dos dados obtidos através da EBIA adaptada, verificou-se um maior percentual de famílias em condição de segurança alimentar SA (18\%) e sem restrição quantitativa de alimentos IAL (50\%) (Figura 1B).

A

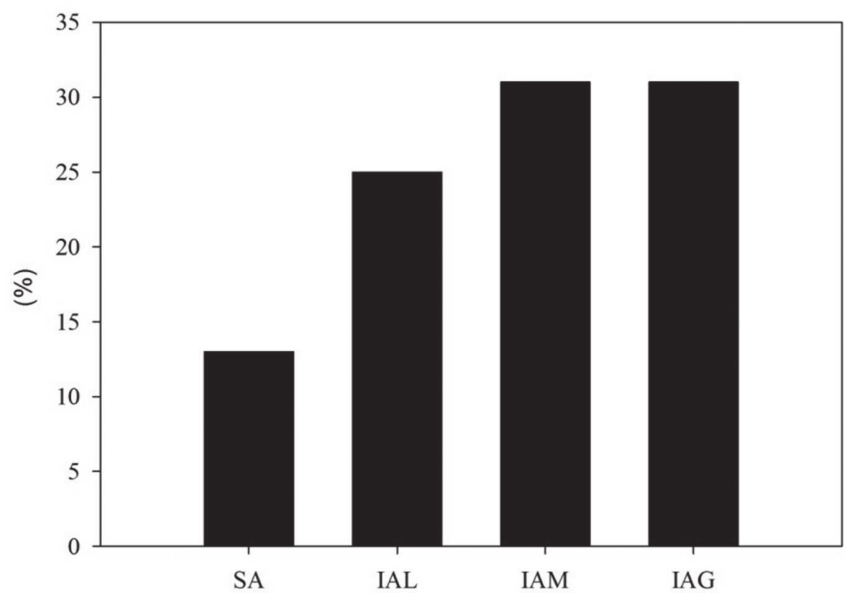

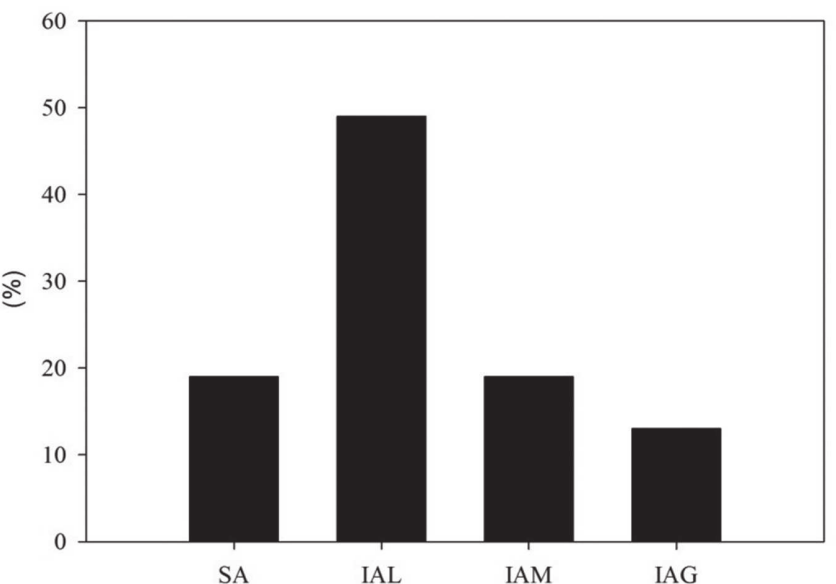

Figura 01 - Gráfico representativo da situação de segurança alimentar dos agricultores com todos os moradores maiores de 18 anos de idade, através da EBIA(A) e a EBIA com escala modificada (B), do município de Tauá, sendo os níveis representados por SA - segurança alimentar; IAL - insegurança alimentar leve; IAM -insegurança alimentar moderada; IAG - insegurança alimentar grave.

Fonte: Autoras.

Para as famílias cujos membros eram todos maiores de 18 anos, a aplicação da EBIA revelou que a insegurança alimentar atingiu todas as famílias com proporções similares de insegurança alimentar leve, moderada e grave $( \pm 33 \%)$ (Figura 2A). Quando aplicada a EBIA adaptada, os resultados foram diferentes, $20 \%$ das 
famílias estavam em condição de segurança alimentar e $80 \%$ das famílias não apresentaram restrições quantitativas de alimentos. (Figura 2B).

A

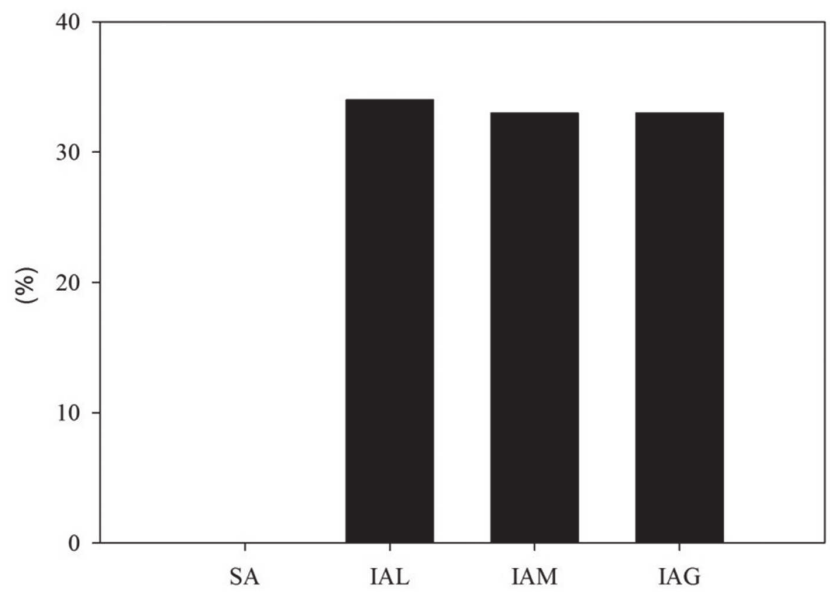

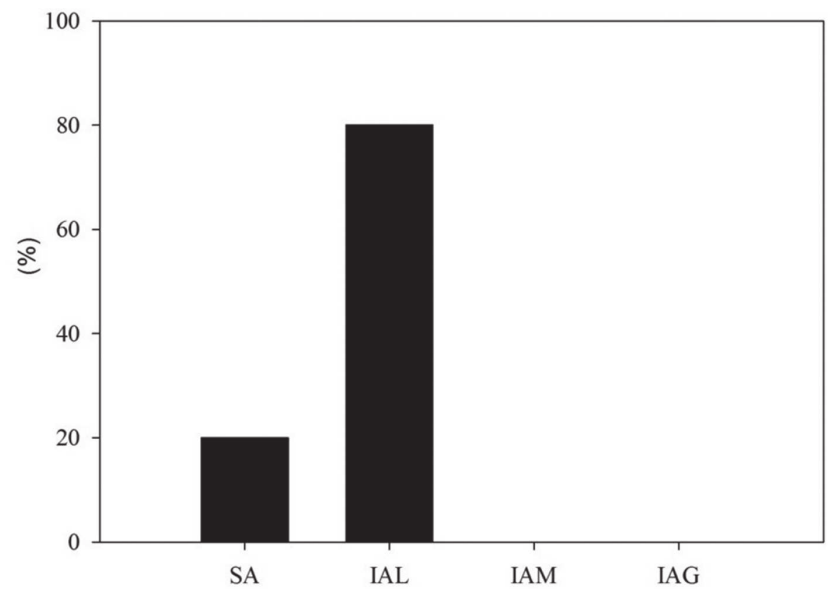

Figura 02 - Gráfico representativo da situação de segurança alimentar dos agricultores com todos os moradores maiores de 18 anos de idade, através da EBIA(A) e a EBIA com escala modificada (B), do município de Tauá, sendo os níveis representados por SA - segurança alimentar; IAL - insegurança alimentar leve; IAM - insegurança alimentar moderada; IAG - insegurança alimentar grave.

Fonte: Autoras.

A aplicação da EBIA no município de Massapê revelou que as famílias dos agricultores com ao menos um morador com menos de 18 anos de idade, encontraram-se em situação de insegurança alimentar, com predomínio da condição de insegurança alimentar leve (79\%) (Figura 3A). Em relação aos valores obtidos com a aplicação da EBIA adaptada, verificou-se que $14 \%$ das famílias estão em condição de segurança alimentar e as demais categorias obtiveram proporções similares ( $\pm 29 \%)$ (Figura 3B).

A

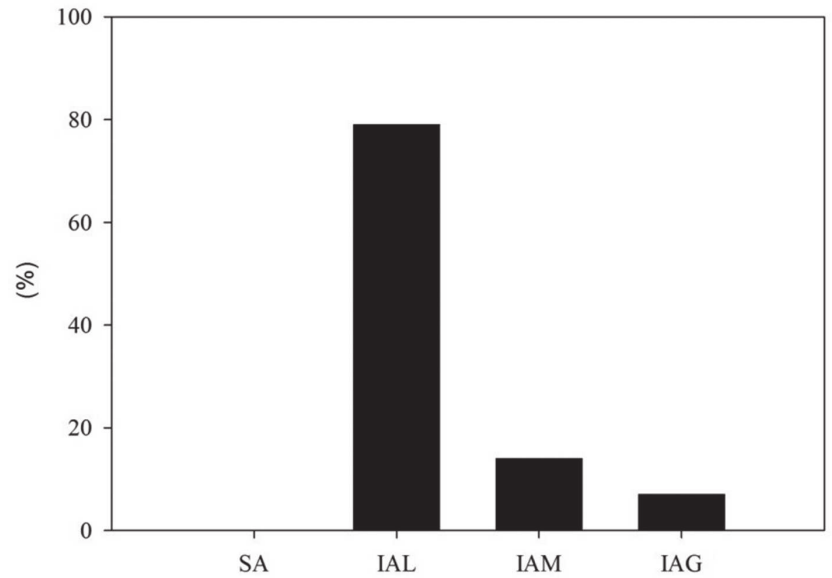

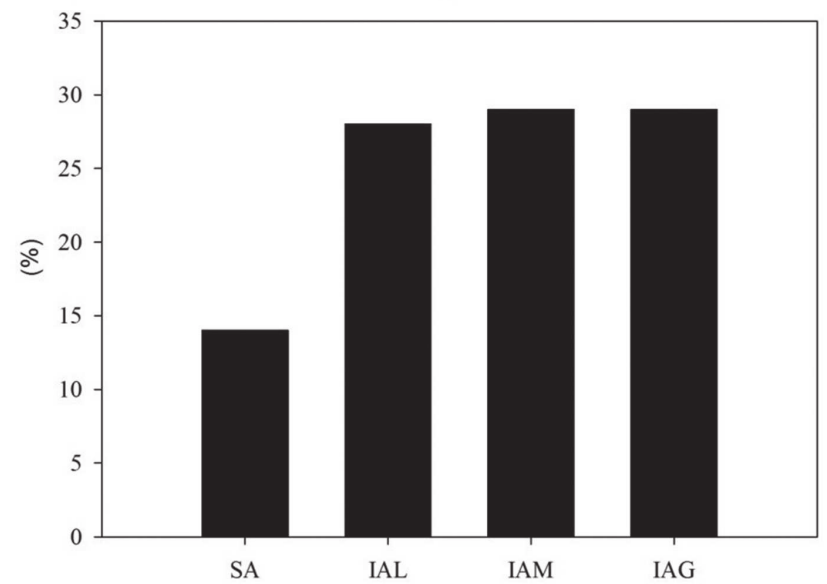

Figura 03 - Gráfico representativo da situação de segurança alimentar dos agricultores entrevistados com ao menos um morador com menos de 18 anos de idade, através da EBIA (A) e a EBIA com escala modificada (B), do município de Massapê, sendo os níveis representados por SA - segurança alimentar; IAL - insegurança alimentar leve; IAM - insegurança alimentar moderada; IAG - insegurança alimentar grave.

Fonte: Autoras. 
Para as famílias dos agricultores de Massapê com todos os seus membros maiores de 18 anos de idade, prevaleceu à condição de insegurança alimentar leve, quando avaliados a partir da EBIA (Figura 4A). A classificação através da EBIA adaptada revelou que as famílias dos agricultores encontravam-se em situação de insegurança alimentar, com proporções similares entre as categorias leve, moderada e grave $( \pm 33 \%)$ (Figura 4B). Esses resultados podem estar relacionados à dinâmica da produção desses agricultores, autoconsumo e comercialização dos itens, ou mesmo a eventos de seca, com prejuízo das colheitas, o que os tornam mais dependentes de renda monetária para compra de alimentos.

A

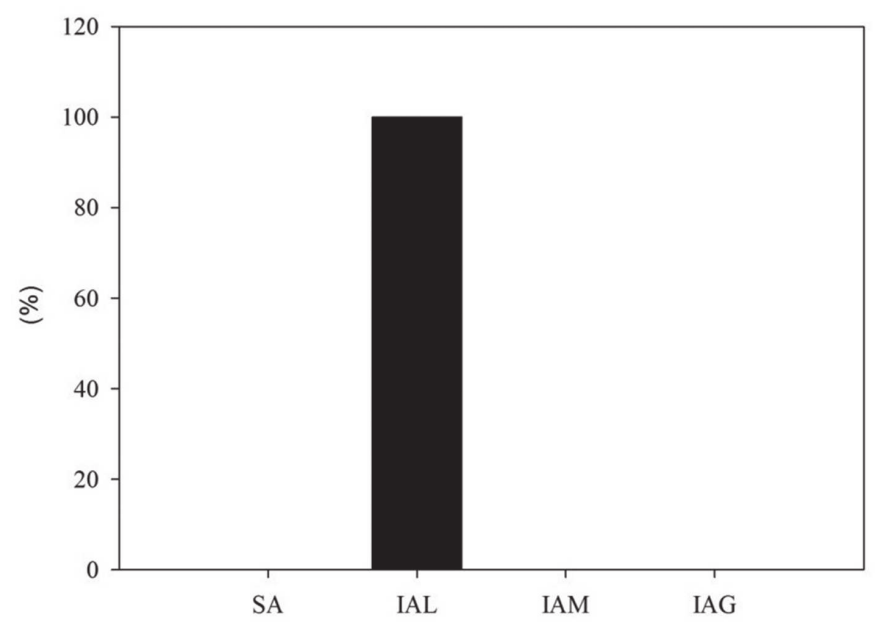

B

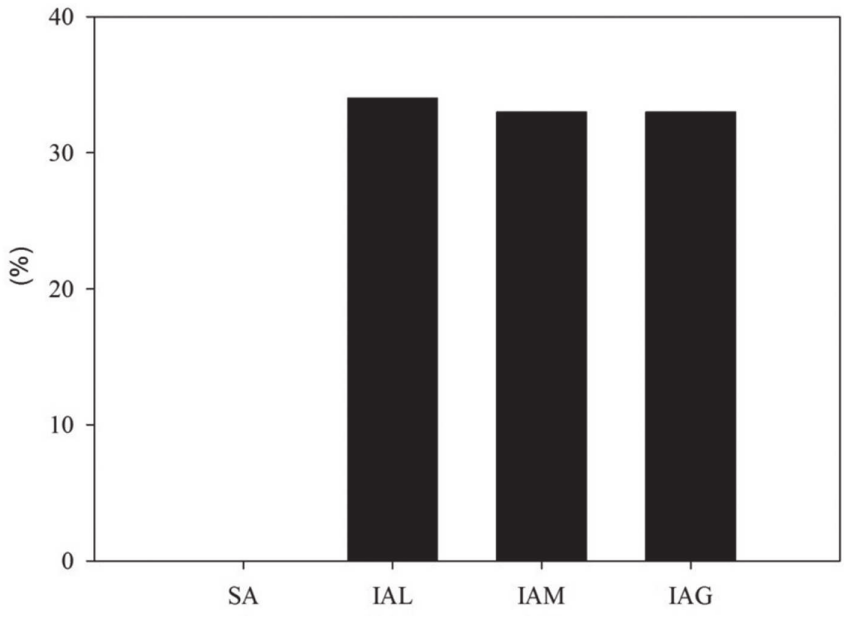

Figura 04 - Gráfico representativo da situação de segurança alimentar dos agricultores entrevistados com todos os moradores maiores de 18 anos de idade, através da EBIA (A) e a EBIA com escala modificada (B), do município de Massapê, sendo os níveis representados por SA - segurança alimentar; IAL - insegurança alimentar leve; IAM - insegurança alimentar moderada; IAG - insegurança alimentar grave.

Fonte: Autoras.

No município de Tauá, quando analisadas através da EBIA, para as famílias com ao menos um morador com menos de 18 anos, apenas 5\% das famílias de agricultores foram classificadas na categoria de segurança alimentar, as categorias insegurança alimentar leve e moderada apresentaram as maiores proporções (33\%), seguida da insegurança alimentar grave (29\%) (Figura 5A). Quando utilizou-se a EBIA adaptada, houve aumento no percentual de famílias em condição de segurança alimentar e que não apresentaram restrição quantitativa de alimentos (Figura 5B). 
A

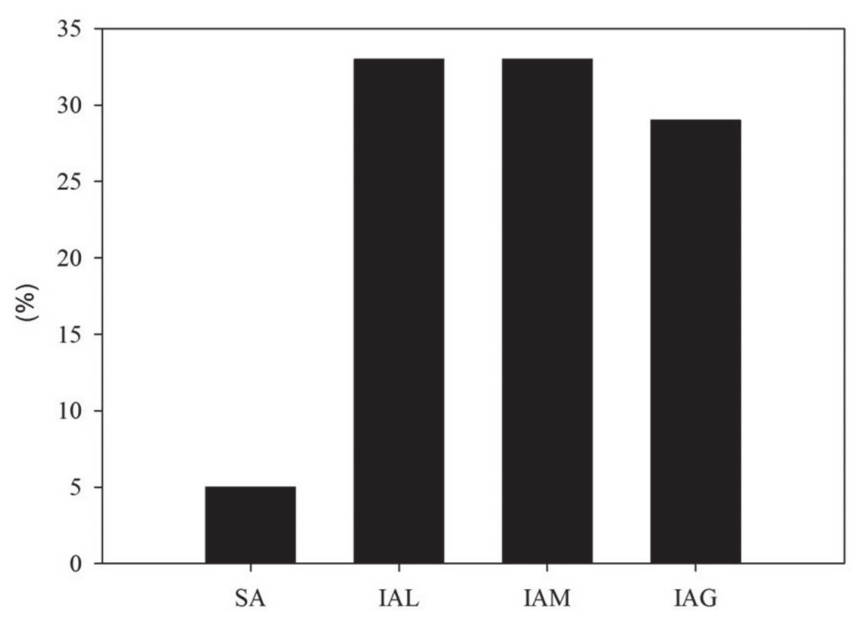

B

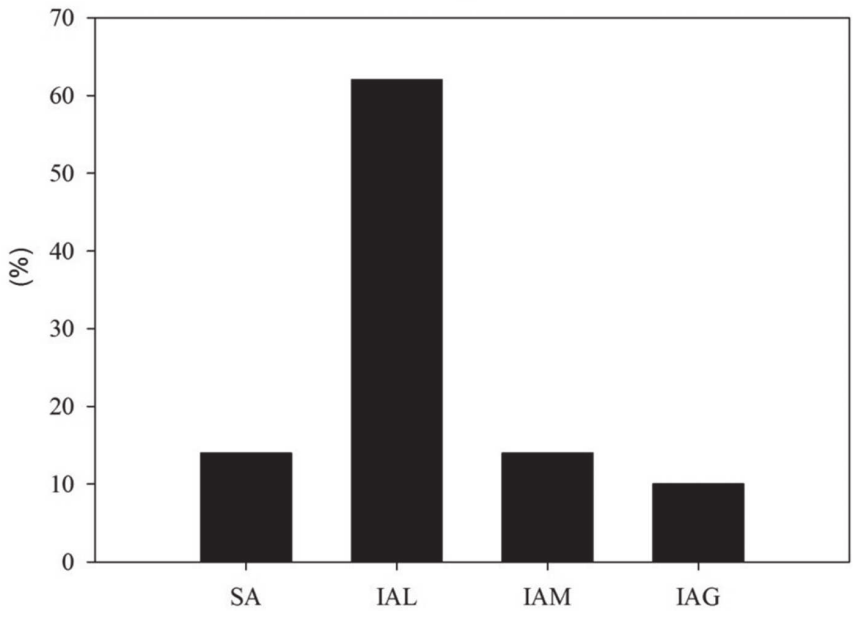

Figura 05 - Gráfico representativo da situação de segurança alimentar dos agricultores entrevistados com ao menos um morador com menos de 18 anos de idade, através da EBIA (A) e a EBIA com escala modificada (B), do município de Tauá, sendo os níveis representados por SA - segurança alimentar; IAL - insegurança alimentar leve; IAM-insegurança alimentar moderada; IAG-insegurança alimenta grave.

Fonte: Autoras.

Em relação às famílias de agricultores desse município, com todos os membros maiores de 18 anos, quando aplicada a EBIA, a condição de insegurança alimentar esteve presente em todas as classificações, com predomínio das categorias de insegurança alimentar leve e grave (40\%) (Figura 6A). Na classificação dessas famílias através da EBIA adaptada, 20\% das famílias encontravam-se em condição de segurança alimentar e $60 \%$ delas não apresentaram restrição quantitativa de alimentos (Figura 6B). Tais resultados evidenciam a importância da produção para o autoconsumo para a segurança alimentar, a qual possibilita o acesso direto aos alimentos.

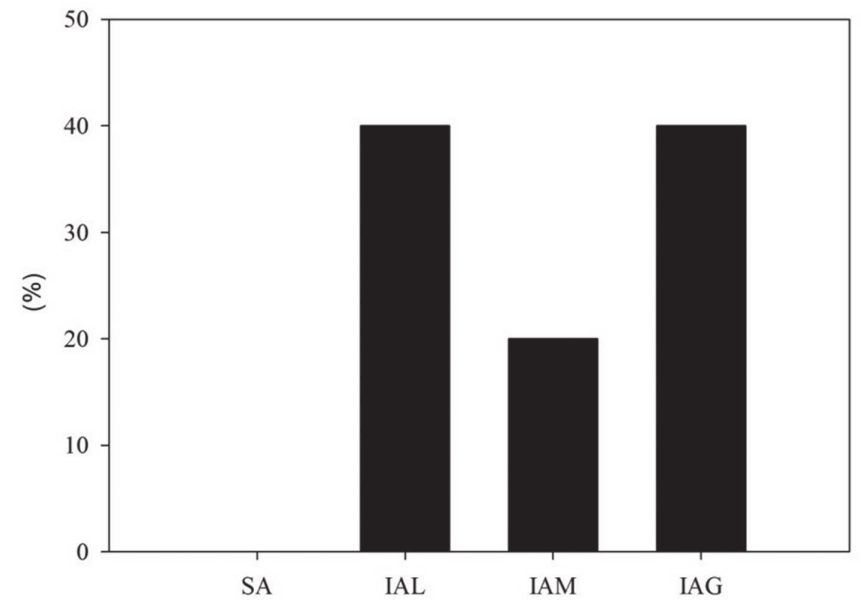

B

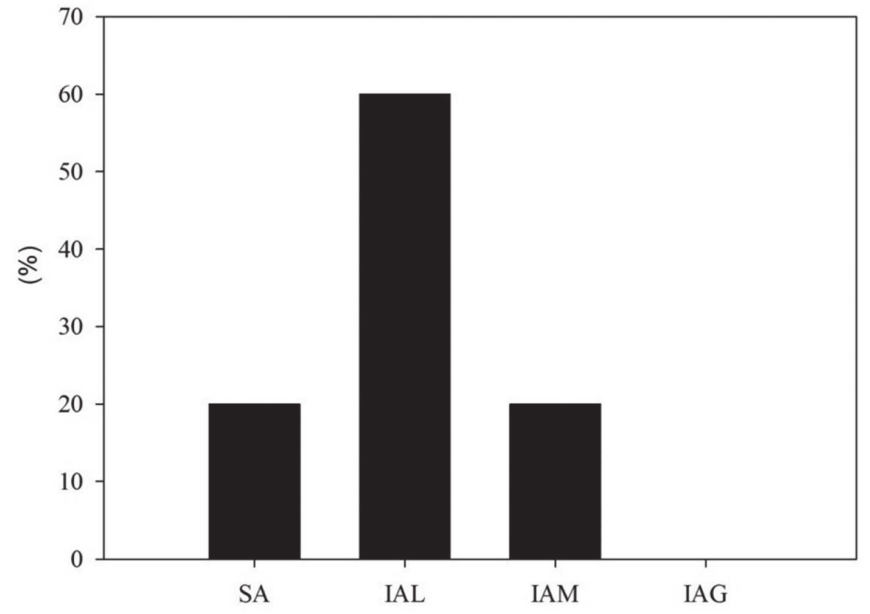

Figura 06 - Gráfico representativo da situação de segurança alimentar dos agricultores com todos os moradores maiores de 18 anos de idade, através da EBIA(A) e a EBIA com escala modificada (B), do município de Tauá, sendo os níveis representados por SA - segurança alimentar; IAL - insegurança alimentar leve; IAM -insegurança alimentar moderada; IAG-insegurança alimenta grave.

Fonte: Autoras. 
De acordo com a EBIA e a EBIA adaptada, as famílias dos municípios de Choró, Massapê e Tauá, foram classificadas em diferentes categorias com e sem membros menores de 18 anos de idade (Figuras 1,2, 3, 4, 5 e 6). Segundo dados do IBGE (2006), a presença de pessoas com menos de 18 anos de idade no domicílio aumenta a insegurança alimentar. No entanto, controlando o efeito da renda domiciliar per capita e de outros fatores, a insegurança alimentar tende a ser menor nos domicílios com pessoas menores de 18 anos (HOFFMANN, 2008). Neste estudo não ficou claro a prevalência da segurança alimentar quando se considera a idade.

Com a exceção do município de Massapê com todos os membros maiores de 18 anos, a escala adaptada demonstrou que a maioria das famílias não apresentaram restrições quantitativas de alimentos em relação à EBIA (Figura 1, 2, 3, 4, 5 e 6). A prevalência da insegurança alimentar sobre a segurança alimentar em todas as famílias através da EBIA pode ser explicada pelo fato dessa escala não incluir o valor da produção para autoconsumo (Tabela 2).

Observa-se, a partir da aplicação da escala adaptada, que as condições precárias da renda que implicaram em insegurança alimentar das famílias analisadas através da EBIA, foram compensadas pelo abastecimento de alimentos produzidos pelos próprios agricultores, os quais exploram a diversidade dos sistemas agroecológicos.

A agroecologia pode melhorar a segurança alimentar através do aumento e conservação da agrobiodiversidade, dos recursos do solo e da água em centenas de comunidades rurais nos países em desenvolvimento (ALTIERI, 2012; KANGMENNAANG et al., 2017). No entanto, a idade, nível educacional, tamanho da família, conhecimento das melhores práticas agrícolas, tamanho da propriedade, cultivo de rendimento, gênero, etnia, renda domiciliar per capta, dentre outros, influenciam nos vários determinantes da insegurança alimentar e nutricional. E grande parte dessas variáveis depende da atuação do Estado (HOFFMANN, 2008).

Um maior número das famílias dos agricultores agroecológicos do município de Tauá (61,8\%) vivia com renda mensal per capita inferior a 50\% da linha de pobreza, ou seja, com renda mensal inferior a meio salário mínimo per capita (IPECE, 2009). Em Massapé e Choró esse percentual foi, respectivamente, de 41\% e 35\% das famílias nessa condição (Tabela 5). Entretanto, no município de Massapê apenas 7\% das famílias de agricultores com ao menos um morador com menos de 18 anos estava em situação de insegurança alimentar grave e nenhuma família com todos os moradores maiores de 18 anos foi classificada nessa categoria, quando avaliadas a partir da EBIA. Nesse caso, Massapê, apresentou a melhor situação quando avaliado através da EBIA.

É possível que a maior renda mensal dos agricultores desse município possa ter influenciado nesse resultado (Tabela 5), tendo em vista que o enfoque da EBIAé o fator renda como determinante da situação de segurança alimentar, o que, segundo Hoffmann (2008), é o fator mais importante para a insegurança alimentar. 
Tabela 5 - Renda mensal e per capita mensal das famílias de agricultores dos municípios de Choró, Massapê e Tauá, 2007.

\begin{tabular}{|c|c|c|c|}
\hline \multirow{2}{*}{ Renda e tamanho médio das famílias } & \multicolumn{3}{|c|}{ Municípios estudados } \\
\hline & Choró & Massapê & Tauá \\
\hline Renda familiar mensal em salário mínimo & & & \\
\hline Menos de 1 salário mínimo & $20 \%$ & $11,7 \%$ & $53,8 \%$ \\
\hline 1 salário mínimo & $15 \%$ & $29,4 \%$ & $8 \%$ \\
\hline Entre 1 e 3 salários mínimos & $65 \%$ & $59 \%$ & 38,2 \\
\hline Mais de 4 salários mínimos & - & - & - \\
\hline Renda per capta & & & \\
\hline Menos de 1/4 salário mínimo & $35 \%$ & $41 \%$ & $61,8 \%$ \\
\hline De $1 / 4$ a $1 / 2$ salário mínimo & $65 \%$ & $59 \%$ & $38,2 \%$ \\
\hline Mais de $1 / 2$ salário mínimo & - & - & - \\
\hline Média do número de pessoas por família & 6 & 6 & 5 \\
\hline
\end{tabular}

Fonte: Dados de pesquisa.

Esses dados permitem inferir que os agricultores agroecológicos dos municípios de Choró e Tauá tem garantido o acesso à alimentação por meio da produção para o autoconsumo e/ou comercialização. Bennett e Franzel (2012) revelaram que, entre 31 casos documentados de agricultores africanos e latino-americanos que converteram sistemas convencionais ou orgânicos, em agricultura orgânica e de conservação de recursos (ORCA - que é um tipo de agricultura que visa à sustentabilidade em longo prazo, como a agricultura agroecológica), os rendimentos melhoraram em $76 \%$ dos casos, a segurança alimentar melhorou em $88 \%$ dos casos e o lucro líquido melhorou em $83 \%$ dos casos.

A insegurança alimentar nos três municípios, quando avaliada a partir da escala adaptada da EBIA, se apresentou com maiores índices na forma de insegurança alimentar leve, caracterizada pela preocupação ou incerteza quanto ao acesso aos alimentos. O que certamente está relacionado à imprevisibilidade climática e consequentemente da produção. Segundo Reganold e Wachter (2016), os usos de estratégias agroecológicas, como a incorporação de resíduos de animais e plantas nos solos, utilizado nas comunidades rurais do presente estudo, podem ajudar a melhorar a fertilidade do solo e a criar resiliência contra a variabilidade climática e a degradação ambiental. Esses métodos agroecológicos reduzem os custos de produção, aumentam a produtividade agrícola, a estabilidade da produção e a resiliência dos agricultores familiares, além de trazer muitos benefícios ao ecossistema (PONÍSIO et al., 2015).

A estratégia dos consórcios agroecológicos desses agricultores ao consorciar o algodão com culturas alimentares (milho, feijão, gergelim) é disponibilizar mais de uma fonte alimentar e de renda, preservar níveis mínimos de segurança alimentar das famílias e dos animais. As culturas alimentares são utilizadas para o consumo 
familiar e o excedente é comercializado, garantindo a complementariedade do consumo. O algodão é a principal cultura geradora de renda, produzido em bases agroecológicas e atinge preços maiores que o algodão convencional. Outra vantagem são os resíduos dessa cultura que servem para alimentação animal.

Outra cultura importante para geração de receita é o gergelim, pela alta produtividade e interesse comercial. Processado, de forma artesanal, nos municípios de Choró e Tauá, o gergelim é transformando em óleo, paçoca, gersal, tahine e doces para consumo próprio e/ou venda nos mercados locais. Dessa forma, agrega mais renda às famílias (CARDOSO, 2017) e amplia o espaço de comercialização de seus produtos.

Desse modo, esses sistemas de produção de base agroecológica, através da diversificação de produtos, têm a possibilidade de produzir alimentos para o autoconsumo, aumentando a autonomia alimentar e geração de renda familiar, ampliando a segurança alimentar e nutricional no meio rural, além da ampliação das relações sociais para fortalecer o grupo dos agricultores envolvidos.

De acordo com Almeida et al. (2015), com a diversidade de renda, pluriatividade econômica, autoconsumo familiar e participação relevante em alguma organização social, o agricultor familiar pode alcançar ou melhorar a sua segurança alimentar. Construir organizações fortes, incorporar sistemas de aquisição e disseminação de conhecimento nas comunidades rurais, a exemplo da ONG ESPLAR. Isso pode ajudar os pequenos agricultores tanto em termos de segurança alimentar quanto em termos de renda para as famílias dos agricultores familiares.

\section{CONCLUSÕES}

As escalas apresentaram resultados divergentes para a situação de segurança alimentar entre os municípios e entre as famílias com ao menos um morador com menos de 18 anos de idade e com todos os moradores maiores de 18 anos de idade. Além da renda, a produção para o autoconsumo demonstrou ser fator determinante da situação de segurança alimentar desses agricultores.

A verificação da insegurança alimentar pela EBIA é superestimada por não considerar a produção para o autoconsumo.

Os métodos agroecológicos combinados com a troca de conhecimentos entre os agricultores e a ONG ESPLAR podem garantir a segurança alimentar.

Programas do governo que têm como estratégia aumentar a renda dos agricultores e de elevar a oferta de produtos agropecuários destinados à alimentação precisam ser mais disseminados e contar com mais recursos, visto que grande parte das famílias em condições de miséria e pobreza no Brasil são agricultores familiares moradores da região do Semiárido.

\section{AGRADECIMENTOS}

Os autores do trabalho agradecem ao CNPq, CAPES e Embrapa Café pelas bolsas dos autores e ao CNPq pelo suporte financeiro por meio da concessão de recursos do Edital CT - Agro/MCT/MDA/CNPq 22/ 
2004, processo 506070-2004 8, no período de novembro de 2004 a outubro de 2007. Os autores agradecem também aos agricultores familiares que colaboraram com essa pesquisa e a todos da equipe ONG ESPLAR.

\section{REFERÊNCIAS}

ABOABA, Kazeem; FADIJI, Damilare Michael; HUSSAYN, Jamiu Ayomide. Determinants of food security among rural households in Nigeria: USDA food insecurity experience based measurement (forms) approach. Journal of Agribusiness and Rural Development, v. 56, n. 2, p. 113-124, 2020.

AGUIAR, Maria Ivanilda. Qualidade física do solo em sistemas agroflorestais. Dissertação (Mestrado em Solos e Nutrição de Plantas) - Universidade Federal de Viçosa, p.79, 2008.

ALAIMO, Katherine; OLSON, Christine M.; FRONGILLO, Edward A. Food insufficiency and American school-aged children's cognitive, academic, and psychosocial development. Pediatrics, v. 108, n. 1, p. 44-51, 2001.

ALMEIDA, Luiz Manoel de Moraes Camargo; PAULILLO, Luiz Fernando Oriani; MAIORANO, Alexandre Cristovão; LOUZADA, Francisco. Índice UFSCar de segurança alimentar para a agricultura familiar. Revista de Política Agrícola, Ano XXIV, n. 4, p. 85-86, 2015.

ALTIERI, Miguel A. Agroecologia, agricultura camponesa e soberania alimentar. Revista nera, n. 16, v. 13, p. 22-32, 2012.

BENNETT, Mica; FRANZEL, Steven. Can organic and resource-conserving agriculture improve livelihoods? A synthesis. International journal of agricultural sustainability, v. 11, n. 3, p. 193-215, 2013.

BHATTACHARYA, Jayanta; CURRIE, Janet; HAIDER, Steven. Poverty, food insecurity, and nutritional outcomes in children and adults. Journal of health economics, v. 23, n. 4, p. 839-862, 2004.

BICKEL, Gary; NORD, Mark; PRICE, Cristofer; HAMILTON, William; COOK, John. Measuring food security in the United States: guide to measuring household food security. Alexandria: U.S. Department of Agriculture, 2000.

BOAS, Lucas Guedes Vilas. Segurança alimentar no campo e na cidade em Nepomuceno-MG. Revista Tamoios, v. 13, n. 1, p. 50-71, 2017.

BORGES, Bárbara Loureiro. Reforma agrária e abastecimento alimentar: a agricultura camponesa do Distrito Federal construindo a segurança e soberania alimentar. 2018. 170f. Dissertação (Mestrado em Meio Ambiente e Desenvolvimento Rural) - Faculdade UNB, Planaltina, 2018.

BORJAS, George. 2002. Food Insecurity and Public Assistance, NBER Working Paper 9236, NBER, Cambridge MA.

BRASIL. Lei n ${ }^{\circ}$ 11.346, de 15 de setembro de 2006. Cria o Sistema Nacional de Segurança Alimentar e Nutricional - Sisan com vistas em assegurar o direito humano à alimentação adequada e dá outras providências. Diário Oficial da União, setembro de 2006. 
CARDOSO, Nágilla Francielle Silva. Algodão agroecológico no semiárido brasileiro: da produção à comercialização. 2017. 84 f. Dissertação (Mestrado em Agroecologia) - Universidade Federal de Viçosa, Viçosa. 2017.

GLIESSMAN, Steve; TITTONELL, Pablo. Agroecology for food security and nutrition, v. 39, n. 2, p. 131$133,2015$.

GRISA, Catia. Segurança alimentar e autonomia na agricultura familiar: a contribuição da produção para o autoconsumo. Revista de extensão e estudos rurais, v.1, n. 1, p. 97-129, 2011.

HOFFMANN, Rodolfo. Determinantes da insegurança alimentar no Brasil: Análise dos dados da PNAD de 2004. Segurança alimentar e nutricional, v. 15, n. 1, p. 49-61, 2008.

HOFFMAN, Rodolfo. Pobreza, insegurança alimentar e desnutrição no Brasil. Estudos Avançados, v. 9 n. 24, 1995.

IBGE-INSTITUTO BRASILEIRO DE GEOGRAFIAEESTATÍSTICA. 2006. Pesquisa Nacional por Amostra de Domicílios. Segurança alimentar 2004. Coordenação de trabalho e rendimento. Rio de Janeiro. 148p.

IBGE - INSTITUTO BRASILEIRO DE GEOGRAFIAEESTATÍSTICA. 2014. Pesquisa Nacional por Amostra de Domicílios: Segurança alimentar 2013. Coordenação de trabalho e rendimento. Rio de Janeiro. 134p.

INMET - INSTITUTO NACIONAL DE METEOROLOGIA. 2016. Dados pluviométricos e de temperatura de Tauá. 2015. Disponível em: <http://www.inmet.gov.br/portal/index.php?r=estacoes/ estacoesAutomaticas>. Acesso em: 02 Ago. 2020.

IPECE - Instituto de Pesquisa e Estratégia Econômica do Ceará. As principais linhas de pobreza utilizadas no Brasil, Nota Técnica n ${ }^{\circ}$ 38, IPECE, 2009.

IPECE - Instituto de Pesquisa e Estratégia Econômica do Ceará. Perfil básico municipal, Choró. Fortaleza: Governo do Estado do Ceará, 2007. 10p.

KANGMENNAANG, Joseph et al. Impact of a participatory agroecological development project on household wealth and food security in Malawi. Food security, v. 9, n. 3, p. 561-576, 2017.

MAIA, Stoécio Malta Ferreira et al. Combined effect of intercropping and minimum tillage on soil carbon sequestration and organic matter pools in the semiarid region of Brazil. Soil Research, v. 57, n. 3, p. 266-275, 2019.

MCKECHNIE, Rebecca et al. Single-item measure of food insecurity used in the National Health Survey may underestimate prevalence in Australia. Australian and New Zealand Journal of public health, v. 42, n. 4, p. 389-395, 2018.

NASCIMENTO, Shirley G. S. et al. Produção agroecológica e Segurança Alimentar e Nutricional (Brasil). Revista de Ciências Agrárias, 2019, v. 42, n. 1, p. 291-300.

OLIVEIRA, Ulisses Costa de et al. Índice de estado trófico para fósforo do açude Acaraú Mirim, município de Massapê, Ceará, nos anos de 2014 a 2017. In: X Congresso Brasileiro de Gestão Ambiental, IDEAS, Fortaleza, Resumos Expandidos. IDEAS, Fortaleza, 2019, p.1-5. 
OTUTUMI, A. T. et al. Qualidade do solo em sistemas de cultivo agroecológico no município de Tauá - CE. In: OLIVEIRA, T. S.(coord.). Solo e Água: aspectos de uso e manejo com ênfase no semi-árido nordestino. Fortaleza, Departamento de Ciências do Solo, UFC, 2004, p. 1-30.

PALMEIRA, Poliana de Araújo et al. Multisectoral government programs and household food insecurity: evidence from a longitudinal study in the semiarid area of northeast, Brazil. Food Security, 2020, p. 1-14.

PEREIRA, Laura; WYNBERG, Rachel; REIS, Yuna. Agroecology: The future of sustainable farming? Environment, v. 60, n. 4, p. 4-17, 2018.

PONISIO, Lauren et al. Diversification practices reduce organic to conventional yield gap. Proceedings of the Royal Society of London B: Biological Sciences, v. 282, n. 1799, 2015.

RADIMER, Kathy L. et al. Understanding hunger and developing indicators to assess it in women and children. Journal of Nutrition Education, v. 24, n. 1, p. 36-44, 1992.

REGANOLD, John P.; WACHTER, Jonathan M. Organic agriculture in the twenty-first century. Nature Plants, v. 2, n. 15221, 2016.

ROSEGRANT, Mark W.; CLINE, Sarah A. Global food security: challenges and policies. Science, v. 302, p. 1917-1919, 2003.

SEGALL-CORRÊA, Ana Maria; MARIN-LEON, Letícia. A segurança alimentar no Brasil: proposição e usos da escala brasileira de medida da insegurança alimentar (EBIA) de 2003 a 2009. Segurança Alimentar e Nutricional, v. 16, n. 2, p. 1-19, 2009.

WEINREB, Linda et al. Hunger: its impact on children's health and mental health. Pediatrics, v. 110, n. 4, 2002.

WORLD HEALTH ORGANIZATION. Food and Agricultural Organization of the United Nations - FAO. The state of food security and nutrition in the world: Bulding climate resilience for food security and nutrition. FAO, p. 202. CC BY-NC-SA 3.0 IGO Rome, 2018.

WORLD HEALTH ORGANIZATION. Food and Agricultural Organization of the United Nations - FAO. The State of Food Security and Nutrition in the World 2019-Safeguarding against economic slowdowns and downturns'. Rome: FAO, p. 239, 2019.

Submetido em: 26/08/2021. Aceito em: 19/02/2021. 